\title{
Embedding of FRW Cosmology in DGP Scenario with a Non-Minimally Coupled Scalar Field on the Brane
}

\author{
Kourosh Nozari ${ }^{a, b}$ \\ a Centre for Particle Theory, Durham University, South Road, Durham DH1 3LE, UK \\ ${ }^{b}$ Department of Physics, Faculty of Basic Sciences, University of Mazandaran, \\ P. O. Box 47416-1467, Babolsar, IRAN \\ e-mail: kourosh.nozari@durham.ac.uk
}

\begin{abstract}
We construct a DGP inspired braneworld scenario where a scalar field nonminimally coupled to the induced Ricci curvature is present on the brane. We show that this model allows for an embedding of the standard Friedmann cosmology in the sense that the cosmological evolution of the background metric on the brane can be described by the standard Friedmann equation. The relation between our framework and the dark-energy formalism is explored.
\end{abstract}

PACS: 04.50.+h, 98.80.-k

Key Words: Braneworld Cosmology, DGP Scenario, Scalar-Tensor Gravity 


\section{Introduction}

Based on light-curves analysis of several hundreds type Ia supernovae[1,2], observations of the cosmic microwave background radiation by the WMAP satellite [3] and other CMBbased experiments[4,5], it has been revealed that our universe is currently in a period of accelerated expansion. Some authors have attributed this late-time expansion of the universe to an energy component referred to as dark energy. The simplest example in this regard is the cosmological constant itself which provides a model of dark energy. However, it is unfavorable since it requires a huge amount of fine-tuning[6]. Phantom fields[7], quintessence[8] and modification of gravitational theory itself $[9,10]$ are other attempts to explain this late time expansion of the universe. In the spirit of modified gravitational theory, Carroll et al have proposed $R^{-1}$ modification of the usual Einstein-Hilbert action[11]. It was then shown that this term could give rise to accelerating solutions of the field equations without dark energy.

On the other hand, theories of extra spatial dimensions, in which the observed universe is realized as a brane embedded in a higher dimensional spacetime, have attracted a lot of attention in the last few years. In this framework, ordinary matters are trapped on the brane but gravitation propagates through the entire spacetime $[9,12,13]$. The cosmological evolution on the brane is given by an effective Friedmann equation that incorporates the effects of the bulk in a non-trivial manner[14]. From a cosmological view point, one of the importance of brane models lies in the fact that they can provide an alternative scenario to explain the late-time accelerated expansion of the universe.

Theories with extra dimensions usually yield correct Newtonian limit at large distances since the gravitational field is quenched on sub-millimeter transverse scales. This quenching appears either due to finite extension of the transverse dimensions $[12,15]$ or due to sub-millimeter transverse curvature scales induced by negative cosmological constant [13,16-19]. A common feature of these type of models is that they predict deviations from the usual 4-dimensional gravity at short distances. The model proposed by Dvali, Gabadadze and Porrati (DGP) [9] is different in this respect since it predicts deviations from the standard 4-dimensional gravity even over large distances. In this scenario, the transition between four and higher-dimensional gravitational potentials arises due to the presence of both the brane and bulk Einstein terms in the action[20]. In this scenario, the existence of a higher dimensional embedding space allows for the existence of bulk or brane matter which can certainly influence the cosmological evolution on the brane. Even if there is no 4-dimensional Einstein-Hilbert term in the classical theory, such a term should be induced by loop-corrections from matter fields [21]. Generally one can consider 
the effect of an induced gravity term as a quantum correction in any brane-world scenario. A particular form of bulk or brane matter is a scalar field. Scalar fields play an important role both in models of the early universe and late-time acceleration. These scalar fields provide a simple dynamical model for matter fields in a brane-world model. In the context of induced gravity corrections, it is then natural to consider a non-minimal coupling of the scalar field to the intrinsic (Ricci) curvature on the brane that is a function of the field. The resulting theory can be thought of as a generalization of Brans-Dicke type scalartensor gravity in a brane-world context. There are several studies in this direction[22-31]. Some of these studies are concentrated on the bulk scalar field minimally[22-24] or nonminimally[25-27] coupled to the bulk Ricci scalar. Some other authors have studied the minimally[28,29] or non-minimally[30,31] coupled scalar field to the induced Ricci scalar on the brane. However, none of these studies have investigated the possibility of embedding of FRW cosmology in DGP scenario with a 4D non-minimally coupled scalar field on the brane. The purpose of this letter is to do this end.

In this letter, in the spirit of DGP inspired gravity, we study the effect of an induced gravity term which is an arbitrary function of a scalar field on the brane. We present four-dimensional equations on a DGP brane with a scalar field non-minimally coupled to the induced Ricci curvature, embedded in a five-dimensional Minkowski bulk. This is an extension to a braneworld context of scalar-tensor (Brans-Dicke) gravity. We show that our model allows for an embedding of the standard Friedmann cosmology in the sense that the cosmological evolution of the background metric on the brane can entirely be described by the standard Friedmann equation plus total energy conservation on the brane. As original DGP scenario and its minimal extension which support late-time acceleration of the universe[20,29], it is easy to show that our model contains this late time acceleration for a suitable range of non-minimal coupling. However, with non-minimally coupled scalar field on the brane, generally it is harder to achieve accelerated expansion[33]. Only with suitable choice of non-minimal coupling and scalar field potential one can achieve accelerated expansion in this non-minimal model.

We use a prime for differentiation with respect to fifth coordinate except for two cases:

$\alpha^{\prime} \equiv \frac{d \alpha}{d \phi}$ and $V^{\prime} \equiv \frac{d V}{d \phi}$. An overdot denotes differentiation with respect to the comoving time, $t$. 


\section{Induced Gravity with Non-Minimally Coupled Brane- Scalar Field}

The action of the DGP scenario in the presence of a non-minimally coupled scalar field on the brane can be written as follows

$$
S=\int d^{5} x \frac{m_{4}^{3}}{2} \sqrt{-g} \mathcal{R}+\left[\int d^{4} x \sqrt{-q}\left(\frac{m_{3}^{2}}{2} \alpha(\phi) R[q]-\frac{1}{2} q^{\mu \nu} \nabla_{\mu} \phi \nabla_{\nu} \phi-V(\phi)+m_{4}^{3} \bar{K}+\mathcal{L}_{m}\right)\right]_{y=0},
$$

where we have included a general non-minimal coupling $\alpha(\phi)$ in the brane part of the action $^{1} . y$ is coordinate of fifth dimension and we assume brane is located at $y=0 . g_{A B}$ is five dimensional bulk metric with Ricci scalar $\mathcal{R}$, while $q_{\mu \nu}$ is induced metric on the brane with induced Ricci scalar $R . g_{A B}$ and $q_{\mu \nu}$ are related via $q_{\mu \nu}=\delta_{\mu}{ }^{A} \delta_{\nu}{ }^{B} g_{A B} . \bar{K}$ is trace of the mean extrinsic curvature of the brane defined as

$$
\bar{K}_{\mu \nu}=\frac{1}{2} \lim _{\epsilon \rightarrow 0}\left(\left[K_{\mu \nu}\right]_{y=-\epsilon}+\left[K_{\mu \nu}\right]_{y=+\epsilon}\right),
$$

and corresponding term in the action is York-Gibbons-Hawking term[32] (see also [20]). The ordinary matter part of the action is shown by Lagrangian $\mathcal{L}_{m} \equiv \mathcal{L}_{m}\left(q_{\mu \nu}, \psi\right)$ where $\psi$ is matter field and corresponding energy-momentum tensor is

$$
T_{\mu \nu}=-2 \frac{\delta \mathcal{L}_{m}}{\delta q^{\mu \nu}}+q_{\mu \nu} \mathcal{L}_{m}
$$

The pure scalar field Lagrangian, $\mathcal{L}_{\phi}=-\frac{1}{2} q^{\mu \nu} \nabla_{\mu} \phi \nabla_{\nu} \phi-V(\phi)$, yields the following energy-momentum tensor

$$
\tau_{\mu \nu}=\nabla_{\mu} \phi \nabla_{\nu} \phi-\frac{1}{2} q_{\mu \nu}(\nabla \phi)^{2}-q_{\mu \nu} V(\phi)
$$

The Bulk-brane Einstein's equations calculated from action (1) are given by

$$
\begin{gathered}
m_{4}^{3}\left(\mathcal{R}_{A B}-\frac{1}{2} g_{A B} \mathcal{R}\right)+ \\
m_{3}^{2} \delta_{A}{ }^{\mu} \delta_{B}{ }^{\nu}\left[\alpha(\phi)\left(R_{\mu \nu}-\frac{1}{2} q_{\mu \nu} R\right)-\nabla_{\mu} \nabla_{\nu} \alpha(\phi)+q_{\mu \nu} \square^{(4)} \alpha(\phi)\right] \delta(y)=\delta_{A}{ }^{\mu} \delta_{B}{ }^{\nu} \Upsilon_{\mu \nu} \delta(y),
\end{gathered}
$$

where $\square^{(4)}$ is 4-dimensional(brane) d'Alembertian and $\Upsilon_{\mu \nu}=T_{\mu \nu}+\tau_{\mu \nu}$. This relation can be written as follows

$$
m_{4}^{3}\left(\mathcal{R}_{A B}-\frac{1}{2} g_{A B} \mathcal{R}\right)+m_{3}^{2} \alpha(\phi) \delta_{A}{ }^{\mu} \delta_{B}{ }^{\nu}\left(R_{\mu \nu}-\frac{1}{2} q_{\mu \nu} R\right) \delta(y)=\delta_{A}{ }^{\mu} \delta_{B}{ }^{\nu} \mathcal{T}_{\mu \nu} \delta(y)
$$

\footnotetext{
${ }^{1}$ For an interesting discussion on the importance of non-minimal coupling and possible schemes to incorporate it in the formulation of scalar-tensor gravity see [31].
} 
where $\mathcal{T}_{\mu \nu}$ is total energy-momentum on the brane defined as follows

$$
\mathcal{T}_{\mu \nu}=m_{3}^{2} \nabla_{\mu} \nabla_{\nu} \alpha(\phi)-m_{3}^{2} q_{\mu \nu} \square^{(4)} \alpha(\phi)+\Upsilon_{\mu \nu},
$$

From (6) we find

$$
G_{A B}=\mathcal{R}_{A B}-\frac{1}{2} g_{A B} \mathcal{R}=0
$$

and

$$
G_{\mu \nu}=\left(R_{\mu \nu}-\frac{1}{2} q_{\mu \nu} R\right)=\frac{\mathcal{T}_{\mu \nu}}{m_{3}^{2} \alpha(\phi)}
$$

for bulk and brane respectively. The corresponding junction conditions relating the extrinsic curvature to the energy-momentum tensor of the brane, have the following form

$$
\lim _{\epsilon \rightarrow+0}\left[K_{\mu \nu}\right]_{y=-\epsilon}^{y=+\epsilon}=\frac{1}{m_{4}^{3}}\left[\mathcal{T}_{\mu \nu}-\frac{1}{3} q_{\mu \nu} q^{\alpha \beta} \mathcal{T}_{\alpha \beta}\right]_{y=0}-\frac{m_{3}^{2} \alpha(\phi)}{m_{4}^{3}}\left[R_{\mu \nu}-\frac{1}{6} q_{\mu \nu} q^{\alpha \beta} R_{\alpha \beta}\right]_{y=0} .
$$

\section{Embedding of FRW Cosmology}

We start with the following line element to derive cosmological dynamics of our model,

$$
d s^{2}=q_{\mu \nu} d x^{\mu} d x^{\nu}+b^{2}(y, t) d y^{2}=-n^{2}(y, t) d t^{2}+a^{2}(y, t) \gamma_{i j} d x^{i} d x^{j}+b^{2}(y, t) d y^{2} .
$$

In this relation $\gamma_{i j}$ is a maximally symmetric 3 -dimensional metric defined as

$$
\gamma_{i j}=\delta_{i j}+k \frac{x_{i} x_{j}}{1-k r^{2}}
$$

where $k=-1,0,1$ parameterizes the spatial curvature and $r^{2}=x_{i} x^{i}$. We assume that scalar field $\phi$ depends only on the proper cosmic time of the brane. Choosing gauge $b^{2}(y, t)=1$ in Gaussian normal coordinates, the field equations in the bulk are given by (8) with the following Einstein's tensor components

$$
\begin{gathered}
G_{00}=3 n^{2}\left(\frac{\dot{a}^{2}}{n^{2} a^{2}}-\frac{a^{\prime 2}}{a^{2}}-\frac{a^{\prime \prime}}{a}+\frac{k}{a^{2}}\right), \\
G_{i j}=\gamma_{i j} a^{2}\left[\left(\frac{a^{\prime 2}}{a^{2}}-\frac{\dot{a}^{2}}{n^{2} a^{2}}-\frac{k}{a^{2}}\right)+2\left(\frac{a^{\prime \prime}}{a}+\frac{n^{\prime} a^{\prime}}{n a}-\frac{\ddot{a}}{n^{2} a}+\frac{\dot{n} \dot{a}}{n^{3} a}+\frac{n^{\prime \prime}}{2 n}\right)\right] \\
G_{0 y}=3\left(\frac{n^{\prime}}{n} \frac{\dot{a}}{a}-\frac{\dot{a}^{\prime}}{a}\right), \\
G_{y y}=3\left(\frac{a^{\prime 2}}{a^{2}}-\frac{\dot{a}^{2}}{n^{2} a^{2}}-\frac{k}{a^{2}}+\frac{n^{\prime} a^{\prime}}{n a}+\frac{\dot{n} \dot{a}}{n^{3} a}-\frac{\ddot{a}}{n^{2} a}\right) .
\end{gathered}
$$


The field equations on the brane are given by the following equations

$$
\begin{gathered}
G_{00}^{(3)}=3 n^{2}\left(\frac{\dot{a}^{2}}{n^{2} a^{2}}+\frac{k}{a^{2}}\right)=\frac{2}{m_{3}^{2} \alpha(\phi)} \mathcal{T}_{00}, \\
G_{i j}^{(3)}=\gamma_{i j}\left[2\left(\frac{\dot{n} \dot{a}}{n^{3} a}-\frac{\ddot{a}}{n^{2} a}\right)-\left(\frac{\dot{a}^{2}}{n^{2} a^{2}}+\frac{k}{a^{2}}\right)\right]=\frac{2}{m_{3}^{2} \alpha(\phi)} \mathcal{T}_{i j},
\end{gathered}
$$

and scalar field evolution equation

$$
\ddot{\phi}+\left(3 \frac{\dot{a}}{a}-\frac{\dot{n}}{n}\right) \dot{\phi}+n^{2} \frac{d V}{d \phi}-\frac{m_{3}^{2}}{2} n^{2} \alpha^{\prime} R[q]=0,
$$

where Ricci scalar on the brane is given by

$$
R=3 \frac{k}{a^{2}}+\frac{1}{n^{2}}\left[6 \frac{\ddot{a}}{a}+6\left(\frac{\dot{a}}{a}\right)^{2}-6 \frac{\dot{a}}{a} \frac{\dot{m}}{n}\right] .
$$

The other important equation is the continuity equation on the brane. Suppose that ordinary matter on the brane has an ideal fluid form, $T_{\mu \nu}=(\rho+p) u_{\mu} u_{\nu}+p q_{\mu \nu}$. Since $K_{t t}=n n^{\prime}$ and $K_{r r}=-a a^{\prime}$, equation (10) gives the following matching conditions

$$
\begin{aligned}
& \lim _{\epsilon \rightarrow+0}\left[\partial_{y} a\right]_{y=-\epsilon}^{y=+\epsilon}(t)=\frac{m_{3}^{2}}{m_{4}^{3}}\left[\alpha(\phi)\left(\frac{\dot{a}^{2}}{n^{2} a}+\frac{k}{a}\right)\right]_{y=0}-\left[\frac{\left(\rho+\rho_{\phi}\right) a}{3 m_{4}^{3}}\right]_{y=0} . \\
& \lim _{\epsilon \rightarrow+0}\left[\partial_{y} n\right]_{y=-\epsilon}^{y=+\epsilon}(t)=\frac{m_{3}^{2}}{m_{4}^{3}}(2 n)\left[\alpha(\phi)\left(\frac{\ddot{a}}{n^{2} a}-\frac{\dot{a}^{2}}{2 n^{2} a^{2}}-\frac{\dot{n} \dot{a}}{n^{3} a}-\frac{k}{2 a^{2}}\right)\right]_{y=0}+ \\
& \frac{n}{3 m_{4}^{3}}\left[2\left(\rho+\rho_{\phi}\right)+3\left(p+p_{\phi}\right)\right]_{y=0}
\end{aligned}
$$

where energy density and pressure of non-minimally coupled scalar field are given as follows

$$
\begin{gathered}
\rho_{\phi}=\left[\frac{1}{2} \dot{\phi}^{2}+n^{2} V(\phi)-6 \alpha^{\prime} H \dot{\phi}\right]_{y=0}, \\
p_{\phi}=\left[\frac{1}{2 n^{2}} \dot{\phi}^{2}-V(\phi)+\frac{2 \alpha^{\prime}}{n^{2}}\left(\ddot{\phi}-\frac{\dot{n}}{n} \dot{\phi}\right)+4 \alpha^{\prime} \frac{H}{n^{2}} \dot{\phi}+\frac{2 \alpha^{\prime \prime}}{n^{2}} \dot{\phi}^{2}\right]_{y=0},
\end{gathered}
$$

and $H=\frac{\dot{a}}{a}$ is Hubble parameter. Note that part of the effect of non-minimal coupling of the field $\phi$ is hidden in the definition of the effective energy density and pressure which both include non-minimal terms. Now using (15) since in the bulk $G_{00}=0$, we find

$$
\lim _{\epsilon \rightarrow+0}\left[\frac{n^{\prime}}{n}\right]_{y=-\epsilon}^{y=+\epsilon}=\left[\frac{\dot{a}^{\prime}}{\dot{a}}\right]_{y=-\epsilon}^{y=+\epsilon}
$$


using relations (21) and (22) we find the following relation for conservation of energy on the brane

$$
\dot{\rho}+\dot{\rho}_{\phi}+3 H\left(\rho+\rho_{\phi}+p+p_{\phi}\right)=6 \alpha^{\prime} \dot{\phi}\left(H^{2}+\frac{k}{a^{2}}\right) .
$$

Thus the non-minimal coupling of the scalar field to the Ricci curvature on the brane through $\alpha(\phi)$ leads to the non-conservation of the effective energy density.

To obtain the cosmological dynamics, we set $n(0, t)=1$. With this gauge condition we recover usual time on the brane via transformation $t=\int^{t} n(0, \eta) d \eta$. In this situation, our basic dynamical variable is only $a(y, t)$ since $n(y, t)$ now is given by

$$
n(y, t)=\frac{\dot{a}(y, t)}{\dot{a}(0, t)} .
$$

where $H=\frac{\dot{a}(0, t)}{a(0, t)}$ is Hubble parameter on the brane. Now we can write the basic set of cosmological equations for a FRW brane in the presence of a non-minimally coupled scalar field. The first of these equations is given by matching condition

$$
\lim _{\epsilon \rightarrow+0}\left[\partial_{y} a\right]_{y=-\epsilon}^{y=+\epsilon}(t)=\frac{m_{3}^{2}}{m_{4}^{3}}\left[\alpha(\phi)\left(\frac{\dot{a}^{2}}{n^{2} a}+\frac{k}{a}\right)\right]_{y=0}-\left[\frac{\left(\rho+\rho_{\phi}\right) a}{3 m_{4}^{3}}\right]_{y=0} .
$$

Insertion of $\frac{n^{\prime}}{n}=\frac{\dot{a}^{\prime}}{\dot{a}}$ into equations (13) and (16) yields the Binétruy et al [14] integral

$$
\mathcal{I}^{+}=\left[\left(\frac{\dot{a}^{2}}{n^{2}}-a^{\prime 2}+k\right) a^{2}\right]_{y>0}
$$

and

$$
\mathcal{I}^{-}=\left[\left(\frac{\dot{a}^{2}}{n^{2}}-a^{\prime 2}+k\right) a^{2}\right]_{y<0},
$$

which are constant and if $a^{\prime}$ is continuous on the brane then $\mathcal{I}^{+}=\mathcal{I}^{-}$. These equations along with scalar field equation

$$
\ddot{\phi}+\left(3 \frac{\dot{a}}{a}-\frac{\dot{n}}{n}\right) \dot{\phi}+n^{2} \frac{d V}{d \phi}-n^{2} \frac{d \alpha}{d \phi} R[q]=0,
$$

and

$$
n(y, t)=\frac{\dot{a}(y, t)}{\dot{a}(0, t)} .
$$

constitute the basic dynamical equations of our model. In the absence of transverse momentum, $\Upsilon_{0 y}=0$, one has $\mathcal{I}^{+}=\mathcal{I}^{-}$. In fact $\mathcal{I}^{ \pm}$can be considered as initial conditions and these quantities reflect the symmetry across the brane. We first consider the case $\mathcal{I}^{+}=\mathcal{I}^{-}$in which follows. Our cosmological equations on the brane now take the following forms (note that $n(0, t)=1$ )

$$
\frac{\dot{a}^{2}(0, t)+k}{a^{2}(0, t)}=\frac{\left(\rho+\rho_{\phi}\right)}{3 m_{3}^{2} \alpha(\phi)}
$$




$$
\begin{gathered}
\ddot{\phi}+3 \frac{\dot{a}(0, t)}{a(0, t)} \dot{\phi}+\frac{d V(\phi)}{d \phi}=\frac{d \alpha}{d \phi} R[q], \\
\mathcal{I}=\left[\dot{a}^{2}(0, t)-a^{\prime 2}(y, t)+k\right] a^{2}(y, t) \\
n(y, t)=\frac{\dot{a}(y, t)}{\dot{a}(0, t)} .
\end{gathered}
$$

Using equation (35), the scale factor is calculated as follows

$$
a^{2}(y, t)=a^{2}(0, t)+\left[\dot{a}^{2}(0, t)+k\right] y^{2}+2\left[\left(\dot{a}^{2}(0, t)+k\right) a^{2}(0, t)-\mathcal{I}\right]^{\frac{1}{2}} y
$$

and therefore $n(y, t)$ is given by equation (36);

$$
\begin{aligned}
& n(y, t)=\left(a(0, t)+\ddot{a}(0, t) y^{2}+a(0, t) \frac{a(0, t) \ddot{a}(0, t)+\dot{a}^{2}(0, t)+k}{\sqrt{\left(\dot{a}^{2}(0, t)+k\right) a^{2}(0, t)-\mathcal{I}}} y\right) \\
& \times\left[a^{2}(0, t)+\left[\dot{a}^{2}(0, t)+k\right] y^{2}+2\left[\left(\dot{a}^{2}(0, t)+k\right) a^{2}(0, t)-\mathcal{I}\right]^{\frac{1}{2}} y\right]^{\frac{-1}{2}}
\end{aligned}
$$

So, the component of 5-dimensional metric (11) are determined. If we set initial conditions in such a way that $\mathcal{I}=0$, we find the following simple equations for cosmological dynamics

$$
\begin{gathered}
a(y, t)=a(0, t)+\left[\dot{a}^{2}(0, t)+k\right]^{\frac{1}{2}} y, \\
n(y, t)=1+\frac{\ddot{a}(0, t)}{\sqrt{\dot{a}^{2}(0, t)+k}} y .
\end{gathered}
$$

Therefore, our model allows for an embedding of the standard Friedmann cosmology in the sense that the cosmological evolution of the background metric on the brane can be described by the standard Friedmann equation.

So far we have discussed the case $\mathcal{I}^{+}=\mathcal{I}^{-}$with a continuous warp factor across the brane. In the case of $\mathcal{I}^{+} \neq \mathcal{I}^{-}$, there cannot be any symmetry across the brane. In this case the basic set of dynamical equations is provided by equations (28), (29), (30) plus the non-conservation of the effective energy density given by (26). In this case, evolution of the scale factor on the brane is given by elimination of $a^{\prime}(y \longrightarrow \pm 0, t)$ from the following generalized Friedmann equation

$$
\begin{gathered}
\pm\left[\dot{a}^{2}(0, t)+k-a^{-2}(0, t) \mathcal{I}^{+}\right]^{\frac{1}{2}} \mp\left[\dot{a}^{2}(0, t)+k-a^{-2}(0, t) \mathcal{I}^{-}\right]^{\frac{1}{2}} \\
=\alpha(\phi) \frac{m_{3}^{2}}{m_{4}^{3}}\left(\frac{\dot{a}^{2}(0, t)+k}{a(0, t)}\right)-\frac{\left(\rho+\rho_{\phi}\right) a(0, t)}{3 m_{4}^{3}} .
\end{gathered}
$$


This is the most general form of modified Friedmann equation for our non-minimal framework. After determination of $a(0, t)$, since $\mathcal{I}^{ \pm}$are constants, $a(y, t)$ can be calculated from (35). This is the full dynamics of the system. Note that in the case where the right hand side of equation (41) is negative, at least one sign in left hand side should be negative depending on initial conditions. However, the dynamics of the problem does not require symmetry across the brane. Therefore, we have shown the possibility of embedding of FRW cosmology in DGP scenario with a 4D non-minimally coupled scalar field on the brane and equation (41) is the most general form of FRW equation in this embedding. This relation for the case with $\mathcal{I}^{+}=\mathcal{I}^{-} \equiv \mathcal{I}$ and a discontinuous warp factor across the $Z_{2}$ symmetric brane leads to the well-known generalization of Friedmann equation in dark energy formalism. To show this feature, we define for simplicity,

$$
\begin{aligned}
x & \equiv H^{2}+\frac{k}{a^{2}}, \\
b & \equiv \rho+\rho_{\phi}, \\
y & \equiv \alpha(\phi) m_{3}^{2},
\end{aligned}
$$

and

$$
z \equiv m_{4}^{3} .
$$

With these definitions, equation (41) (with upper sign for instance), transforms to the following form

$$
\left(x-\frac{\mathcal{I}^{+}}{a^{4}}\right)^{\frac{1}{2}}+\left(x-\frac{\mathcal{I}^{-}}{a^{4}}\right)^{\frac{1}{2}}=\frac{y}{z} x-\frac{b}{3 z} .
$$

Solving this equation for $x$ (with $\mathcal{I}^{+}=\mathcal{I}^{-} \equiv \mathcal{I}$ ) gives the following result

$$
x=\frac{\frac{b y}{3 z^{2}}+2 \pm \sqrt{\left(\frac{b y}{3 z^{2}}+2\right)^{2}-\frac{y^{2}}{z^{2}}\left(\frac{b^{2}}{9 z^{2}}+\frac{4 \mathcal{I}}{a^{4}}\right)}}{\frac{y^{2}}{z^{2}}} .
$$

A little algebraic manipulation gives

$$
x=\frac{1}{3 y}\left[b+\frac{6 z^{2}}{y} \pm \frac{6 z^{2}}{y} \sqrt{1+\frac{b y}{3 z^{2}}-\frac{\mathcal{I} y^{2}}{a^{4} z^{2}}}\right] .
$$

Considering both plus and minus signs in equation (41) and using original quantities we obtain

$$
H^{2}+\frac{k}{a^{2}}=\frac{1}{3 m_{3}^{2} \alpha(\phi)}\left(\rho+\rho_{\phi}+\rho_{0}\left[1+\varepsilon \sqrt{1+\frac{2}{\rho_{0}}\left[\rho+\rho_{\phi}-m_{3}^{2} \alpha(\phi) \frac{\mathcal{E}_{0}}{a^{4}}\right]}\right) .\right.
$$


where $\rho_{0} \equiv \frac{6 z^{2}}{y}=\frac{6 m_{4}^{6}}{m_{3}^{2} \alpha(\phi)}, \quad \varepsilon= \pm 1$ shows the possibility of existence of two different branches of FRW equation and $\mathcal{E}_{0}=3 \mathcal{I}$ is a constant. This analysis shows the consistency of our formalism with dark-radiation formalism presented in [23,30]. In the high energy regime where $\frac{\rho+\rho_{\phi}}{\rho_{0}} \gg 1$, we find

$$
H^{2}+\frac{k}{a^{2}} \approx \frac{1}{3 m_{3}^{2} \alpha(\phi)}\left(\rho+\rho_{\phi}+\varepsilon \sqrt{2\left(\rho+\rho_{\phi}\right) \rho_{0}}\right)
$$

which describes a four dimensional gravity with a small correction. Neglecting this small correction, the resulting equation is exactly the same as equation (33). In the low energy regime where $\frac{\rho+\rho_{\phi}}{\rho_{0}} \ll 1$, we find

$$
H^{2}+\frac{k}{a^{2}} \approx \frac{1}{3 m_{3}^{2} \alpha(\phi)}\left[(1+\varepsilon)\left(\rho+\rho_{\phi}\right)+(1+\varepsilon) \rho_{0}-\frac{\varepsilon}{4} \frac{\left(\rho+\rho_{\phi}\right)^{2}}{\rho_{0}}\right] .
$$

For $\varepsilon=+1$ this equation describes a four-dimensional gravity, while for $\varepsilon=-1$ we have a five-dimensional gravity.

\section{Summary and Conclusions}

In this paper we have considered the DGP model with a non-minimally coupled scalar field on the brane. The introduction of non-minimal coupling is not just a matter of taste; it is forced upon us in many situations of physical and cosmological interests such as quantum corrections to the scalar field theory and its renormalizability in curved spacetime. In the spirit of DGP inspired gravity, we have studied the effect of an induced gravity term which is an arbitrary function of a scalar field on the brane. We have presented four-dimensional equations on a DGP brane with a scalar field non-minimally coupled to the induced Ricci curvature, embedded in a five-dimensional Minkowski bulk. This is an extension to a braneworld context of scalar-tensor (Brans-Dicke) gravity. Our model does not describe a special dynamics for late time acceleration beyond the standard DGP scenario. In other words, as original DGP scenario and its minimal extension which support late-time acceleration of the universe[20,29], it is easy to show that our model contains this late time acceleration for a suitable range of non-minimal coupling. However, with non-minimally coupled scalar field on the brane, generally it is harder to achieve accelerated expansion[31,33]. Only with suitable choice of non-minimal coupling and scalar field potential one can achieve accelerated expansion in this non-minimal model. The main ingredient of our analysis lies in the fact that DGP model allows for an embedding of the standard Friedmann cosmology in the sense that the cosmological evolution of the background metric on the brane can entirely be described by the standard Friedmann equation 
plus total energy conservation on the brane and the dynamics of the problem does not require symmetry across the brane. Our general framework applied to a $Z_{2}$-symmetric brane gives the well-known result of dark energy formulation.

\section{Acknowledgement}

It is a pleasure to appreciate members of the Centre for Particle Theory at Durham University, specially Professor Ruth Gregory for hospitality. I would like also to appreciate referee for his/her important contributions in this work.

\section{References}

[1] A. G. Riess et. al. (Supernova Search Team Collaboration), Astron. J. 116 (1998) 1006, [astro-ph/9805201].

[2] S. Perlmutter et. al., Astrophys. J. 517 (1999) 565, [astro-ph/9812133],

D. N. Spergel et. al., Astrophys. J. Suppl. 148 (2003) 175, [astro-ph/0302209].

[3] C. L. Bennett et. al., Astrophys. J. Suppl. 148 (2003) 1, [astro-ph/0302207].

[4] C. B. Netterfield et. al., Astrophys. J. 571 (2002) 604, [astro-ph/0104460],

N. W. Halverson et. al., Astrophys. J. 568 (2002) 38, [astro-ph/0104489].

[5] D. N. Spergel et al, WMAP Three Year Results: Implication for Cosmology, arXiv:astro-ph/0603499

[6] T. Padmanabhan, Phys. Rept. 380 (2003) 253

V. Shani and A. A. Astarobinsky, Int. J. Mod. Phys. D 9 (2000) 373

S. M. Carroll, Living Rev. Rel. 4 (2001) 1

E. J. Copeland, M. Sami and S. Tsujikawa, arXiv:hep-th/0603057

S. Nesseris and L. Perivolaropoulos, arXiv:astro-ph/0610092

[7] R. R. Caldwell, Phys. Lett. B 545 (2002) 23

R. R. Caldwell, M. Kamionkowski and N. N. Weinberg, Phys Rev. Lett. 91 (2003) 071301

J. M. Cline, S. Y. Jeon and G. D. Moore, Phys. Rev. D 70 (2004) 043543

[8] R. R. Caldwell, R. Dave and P. J. Steinhardt, Phys. Rev. Lett. 80 (1998) 1582

P. J. E. Peebles and A. Vilenkin, Phys. Rev. D 59 (1999) 063505

P. J. Steinhardt, L. M. Wang and I Zlatev, Phys. Rev. D 59 (1999)123504 
M. Doran and J. Jaechel, Phys. Rev. D 66 (2002) 043519

A. R. Liddle, P. Parson and J. D. Barrow, Phys. Rev. D 50 (1994) 7222

[9] G. Dvali, G. Gabadadze and M. Porrati, Phys. Lett. B 485 (2000) 208, [hepth/0005016]

G. Dvali and G. Gabadadze, Phys. Rev. D 63 (2001) 065007, [hep-th/0008054],

G. Dvali, G. Gabadadze, M. Kolanović and F. Nitti, Phys. Rev. D 65 (2002) 024031, [hep-th/0106058]

[10] C. Deffayet, Phys. Lett. B 502 (2001) 199, [hep-th/0010186]

C. Deffayet, G. R. Dvali and G. Gabadadze, Phys. Rev. D 65 (2002) 044023, [astro$\mathrm{ph} / 0105068]$

C. Deffayet and S. J. Landau, J. Raux, M. Zaldarriaga and P. Astier, Phys. Rev. D 66 (2002) 024019, [astro-ph/0201164],

J. S. Alcaniz, Phys. Rev. D 65 (2002) 123514, [astro-ph/0202492]

D. Jain, A. Dev and J. S. Alcaniz, Phys. Rev. D 66 (2002) 083511, [astro-ph/0206224]

A. Lue, R. Scoccimarro, G. Starrkman, Phys. Rev. D 69 (2004) 044005, [astro$\mathrm{ph} / 0307034]$.

[11] S. M. Carroll, V. Duvvuri, M. Trodden, M. Turner, Phys. Rev. D 70 (2004) 043528, [astro-ph/0306438].

[12] N. Arkani-Hamed, S. Dimopoulos, G. Dvali, Phys. Lett. B 429 (1998) 263, [hep$\mathrm{ph} / 9803315]$

N. Arkani-Hamed, S. Dimopoulos, G. Dvali, Phys. Rev. D 59 (1999) 086004, [hepth/9807344]

[13] L. Randall, R. Sundrum, Phys. Rev. Lett. 83 (1999) 3370

L. Randall, R. Sundrum, Phys. Rev. Lett. 83 (1999) 4690

[14] P. Binétruy, C. Deffayet, D. Langlois, Nucl. Phys. B 565 (2000) 269, [hep-th/9905012] P. Binétruy, C. Deffayet, U. Ellwanger and D. Langlois, Phys. Lett. B 447 (2000) 285, [hep-th/9905012]

R. Maartens, Living Rev. Relativity, 7 (2004) 7, http://www.livingreviews.org/lrr2004-7

D. Wands, arXiv: gr-qc/0601078 and references therein.

[15] I. Antoniadis, N. Arkani-Hamed, S. Dimopoulos, G. Dvali, Phys. Lett. B 436 (1998) 257 
D. Cremades, L. E. Ibanez and F. Marchesano, Nucl. Phys. B 643 (2002) 93, [hepth/0205074]

C. Kokorelis, Nucl. Phys. B 677 (2004) 115, [hep-th/0207234]

[16] W. Mück, K. S. Viswanathan, I. V. Volovich, Phys. Rev. D 62 (2000) 105019, [hepth/0004017].

[17] R. Gregory, V. A. Rubakov, S. M. Sibiryakov, Class. Quan. Grav. 17 (2000) 4437, [hep-th/0003109].

[18] I. Ya. Aref'eva, M. G. Ivanov, W. Mück, K. S. Viswanathan and I. V.Volovich, Nucl. Phys. B 590 (2000) 273, [hep-th/0004114].

[19] M. Cvetič, M. J. Duff, J.T. Liu, H. Lu, C. N. Pope and K. S. Stelle, Nucl. Phys. B 605 (2001) 141, [peh-th/0011167].

[20] R. Dick, Class. Quant. Grav. 18 (2001) R1, [hep-th/0105320]

R. Dick, Actaphys. Polon. B 32 (2001) 3669, [hep-th/0110162].

[21] H. Collins and B. Holdom, Phys. Rev. D 62 (2000) 105009, [arXiv:hep-th/0003173] Y. V. Shtanov, arXiv:hep-th/0005193

N. J. Kim, H. W. Lee and Y. S. myung, Phys. Lett. B 504 (2001) 323, [arXiv:hepth/0101091]

[22] S. Mizuno, Kei-ichi Maeda and K. Yamamoto, Phys. Rev. D 67 (2003) 023516, [hepth/0205292]

[23] Kei-ichi Maeda, S. Mizuno and T. Torii, Phys. Rev. D 68 (2003) 024033, [arXiv:grqc/0303039]

[24] S. C. Davis, JHEP 03 (2002) 058, [arXiv:hep-ph/0111351 and arXiv:hep-th/0106271]

[25] C. Bogdanos, A. Dimitriadis and K. Tamvakis, arXiv:hep-th/0611181

[26] A. Dimitriadis, C. Bogdanos and K. Tamvakis, Phys. Rev. D 74 (2006) 045003

[27] K. Farakos and P. Pasipoularides, Phys. Lett. B 621 (2005) 244

K. Farakos and P. Pasipoularides, Phys. Rev. D 73 (2006) 084012, [arXiv:hepth/0610010].

[28] Rong-Gen Cai and H. Zhang, JCAP 0408 (2004) 017, [arXiv:hep-th/0403234] 
[29] K. Atazadeh and H. R. Sepangi, Phys. Lett. B 643 (2006) 76, [arXiv:gr-qc/0610107]

[30] M. Bouhamdi-Lopez and D. Wands, Phys. Rev. D 71 (2005) 024010,[arXiv:hepth/0408061]

[31] V. Faraoni, Phys. Rev. D 62 (2000) 023504, [arXiv:gr-qc/0002091]

[32] J. W. York, Phys. Rev. Lett. 28 (1972) 1082

G. W. Gibbons and S. W. Hawking, Phys. Rev. D 15 (1977) 2752

[33] V. Faraoni, Phys. Rev. D 53 (1996) 6813 